\title{
Machine Learning Based Approach for Detection of Alzheimer's Disease
}

\author{
Maskeen kaur*and Amanjot kaur** \\ * Master's Student, Baba Banda Singh Bahadur Engineering College, Fatehgarh Sahib (Punjab), India \\ ** Professor (CSE), Baba Banda Singh Bahadur Engineering College, Fatehgarh Sahib (Punjab), India \\ DOI: 10.29322/IJSRP.11.11.2021.p11942 \\ http://dx.doi.org/10.29322/IJSRP.11.11.2021.p11942
}

\begin{abstract}
Alzheimer's disease (AD) is an irreversible, revolutionary neurodegenerative disease that slowly destroys reminiscence and wondering skills and eventually, the capacity to perform the only tasks.In this paper, prediction of AD based on a deep neural network from magnetic resonance imaging (MRI) is proposed.Recognizing signs early as much as possible is important as disorder enhancing drugs might be best if administered early with inside the route of disorder, earlier than the incidence of irreversible brain damages. Therefore, there may be a excessive significance of utilising computerized strategies for the cause of pre-detection of $A D$ signs from such data. The proposed Convolution neural network model shows the accuracy of $82.3 \%$ with the use of the OASIS dataset.The model is smoother, and supports the view that simpler networks are easier to train. A simple, smaller model also can go with the best results. Not only for the detection of Alzheimers disease but also for the other diseases detection as brain tumor, breast cancer or heart failure diseases 3D MRI data plays a very important role as it can come up with the better results for the proper diagnosis of the particular disease.
\end{abstract}

Index Terms- Alzheimer disease, Machine learning, Alzheimer Magnetic Resonance Imaging (MRI) , Convolution Neural network.

\section{INTRODUCTION}

$\mathrm{D}$ ementia is a syndrome due to disease of the brain, usually chronic, characterized by a progressive, global deterioration in intellect including memory, learning, orientation, language, comprehension and judgment (Walokar,2012).

Alzheimer's disease (AD) is a one of the maximum vital instance of dementia. Alzheimer's disease influences humans in a several way. Patients be afflicted by reminiscence loss, confusion, trouble in speaking, analysing or writing. Eventually, they will overlook approximately their existence and couldn't understand even their own circle of relatives members. They can overlook the way to carry out each day sports together with brushing enamel or combing hair. As a result, it makes humans annoying or competitive or to wander far from home. Alzheimer's disease may even motive demise in elder humans. There are 3 primary levels in Alzheimer's ailment - very moderate, moderate and mild. Detection of Alzheimer's disease (AD) remains now no longer correct till the affected person reaches a mild AD.
The first important changes consist of trouble in quick time period memory, disorientation, loss of self-care, behavioural adjustments, depression, anxiety, and issues with language. It is usually widespread that Alzheimer's disease has a sluggish and irreversible development. In the terminal levels of the sickness, the affected person might also additionally lose capacity to perceive, think, talk or move, in the long run main to lack of physical capabilities after which eventually to death. The development of the disease might also additionally take from three to nine years, or maybe longer.

The early detection of Alzheimer's disease still a undertaking due to the estimation of the scans relies upon on guide directing and visible reading. This preclinical degree is likewise called Mild Cognitive Impairment (MCI). As the mind harm progresses, different cognitive deterioration seem and the disease will become obvious. In the overdue stages, men and women are absolutely depending on caregivers even for the maximum simple each day responsibilities including eating, bathing or dressing. Till now, there's no treatment for Alzheimer's disease, however its early detection is vital to a success treatment, slackens the development of symptoms. So, the improvement of automated diagnostic tools, that is reliant on records received from 3-d images of the mind, has attracted splendid interest in closing years. Computer Aided Diagnosis (CAD) permits early detection of the sickness at early stages, and structural mind photographs are beneficial on this task.

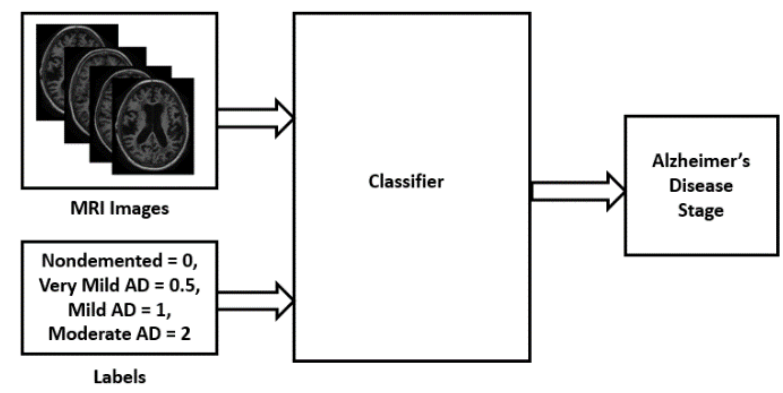

Fig.1:Diagram of a generic Alzheimer's disease detection and classification framework.

The maximum typically used imaging method with the aid of using docs is Magnetic resonance imaging (MRI) which makes use of a magnetic subject and pulses of radio wave power to make images of organs and systems in the body. The number one 
position of MRI with inside the evaluation of AD is the assessment of quantity alteration in characteristic positions, which could provide an analytical accuracy of as much as $87 \%$. In many cases, MRI offers one of a kind data approximately systems withinside the body than may be visible with an X-ray, ultrasound, or computed tomography (CT) scan. Magnetic resonance imaging (MRI) is taken into consideration the favoured neuroimaging exam for Alzheimer disease as it lets in for correct size of the 3dimensional (3D) quantity of brain structures.

Machine learning studies the usage of neuroimaging facts for growing diagnostic gear helped loads for automatic mind MRI segmentation and classification. Most of them use hand-made function generation and extraction from the MRI facts. After that, the capabilities are fed into machine learning models inclusive of Support Vector Machine, Logistic regression model, etc. These multi-step architectures are complicated and extraordinarily depending on human experts. Besides, the dimensions of datasets for neuroimaging research are small. While image classification datasets used for object detection and classification has tens of thousands and thousands of images, neuroimaging datasets generally have much less than a thousand images. But to broaden robust neural networks we want a whole lot of images. Because of the shortage of massive image database, it's far crucial to broaden models that may study beneficial capabilities from the small dataset.

CNNs are mainly used for graphical data in recognition, processing of images. These are inspired by the human visual systems and were created by Yann LeCun (LeCun,1998). The input image is broken down into smaller chunks known as local receptive fields. CNN comes with 3 main layers each having a specific function to perform namely, convolutional layer, pooling layer and fully connected layer. Convolutional layer has set of learnable filters that can be applied on input images.

\section{LITERATURE SURVEY}

Developing an automated Alzheimer 'sdisease detection and classification model is a pretty challenging task. But there is some remarkable research work in this area.Several techniques have been introduced for the diagnosis of AD using image processing and machine learning techniques, which perform more efficiently as compared with manual systems.

Madhumitha et al. (Patil, 2015)offered the usage of image processing on MRI scans. The atrophy is computed via K-means, wavelet, watershed, and some different custom designed algorithms. The outcomes of this method may also offer a useful diagnostic degree for early level AD.

Islam and Zhang (Islam, 2017)provides a unique deep getting to know version for multi-Class Alzheimer's disorder detection and type the usage of brain MRI Data. They layout a completely deep convolutional network and exhibit the overall performance at the Open Access Series of Imaging Studies (OASIS) database. They had tested the overall performance of the version on OASIS dataset. The approach become faster, and it does now no longer want any handmade feature, and it is able to deal with the small clinical photograph dataset. It affords one step evaluation for the brain MRI information for AD detection and type.
Acharya et al. (Acharya, 2019) presented work on the development of a Computer-Aided Brain Diagnosis (CABD) system that can determine whether a brain scan shows signs of Alzheimer's disease. The method uses magnetic resonance imaging (MRI) for classification with various feature extraction techniques. The paradigm consists of a number of quantitative techniques: filtering, feature extraction, feature selection based on Student's t-test, and classification based on nearest neighbour (KNN). It is performed by implementing other feature extraction methods described in the literature. The results suggest that the Shearlet Transform (ST) feature extraction technique provides better results for diagnosing Alzheimer's disease when compared to alternative methods. Delivered94.54\% accuracy, $88 \%$ accuracy.33\%, sensitivity $96.30 \%$ and specificity $93.64 \%$. In addition, this tool with the MRI reference database provided accuracy, precision, sensitivity and specificity of $98.48 \%, 100 \%$, $96.97 \%$ and $100 \%$, respectively.

Toro et al. (Toro,2019)presented an analysis of textural radiomic features extracted from a gray matter probability volume in a number of individual subcortical regions from several different atlases in order to identify patients with $\mathrm{AD}$ on magnetic resonance imaging. Experimental results using the ADNI1 database have shown the potential of some of the radiomic features tested as potential biomarkers for $\mathrm{AD} / \mathrm{CN}$ differentiation. Different traits, both neuromorphometry and Hammers, have a slight advantage over the others as the most suitable atlases for detecting Alzheimer's disease, although the differences in performance are generally small. The results obtained for some of the traits tested suggest extending the study to other cases, such as differentiating between normal and mild cognitive impairment, and expanding the group of study participants using other databases. Also considered is the possibility of narrowing down the amount of subcortical regions used, as well as improving the discrimination of the models using multifactorial vectors.

Ammarah Farooq et al. (Farooq, 2017)presented a deep CNN-based multiclass classification of Alzheimer's disease using magnetic resonance imaging. In this work a 4-way classifier is implemented to classify Alzheimer's disease (AD), mild cognitive impairment (MCI), late mild cognitive impairment (LMCI) and healthy people using an ADNI data set.Theproposed approach outcomes in a prediction accuracy of $98.8 \%$.

$\mathrm{H}$ Allioui et al.(Allioui,2019) presents the methods that automatically perform brain segmentation for the detection of brain damage and the early diagnosis of the Alzheimerdisease. In this method 2.5 D was used for locating brain inflammation and detecting the class. The method was evaluated on the public data. Computational cost and complexity was reduced. The method achieved the accuracy rate of $92.7 \%$.

H M T Ullah et al.(Ullah, 2018) an opportunity method has been discussed, this is fast, expenses much less and extra reliable. Deep Learning represents the authentic bleeding fringe of Machine Intelligence. Convolutional Neural Networks are biologically stimulated Multilayer perceptron specifically able to image processing. In this paper the gift a state of the artwork Deep Convolutional Neural Network to come across Alzheimer's Disease and Dementia from 3-D MRI picture.

Al-falluji and Abdulmunem (Al-falluji, 2017)conducted the survey on magnetic resonance-based techniques for detecting Alzheimer's disease. The article presents an overview of various 
classifier and feature extraction algorithms used to design the Alzheimer's CAD system. They are calculated by different authors using different techniques to identify or calculate the course of Alzheimer's disease, which in the future will need to be compared with other imaging methods to see which method offers greater accuracy

Khan and Usman (Khan, 2015)present an overview, analysis, and critical assessment of recent work on the early detection of $\mathrm{AD}$ with ML techniques. Several methods have shown promising predictive accuracies; however, they have been evaluated on different pathologically unproven data sets from different imaging modalities, making a fair comparison between them difficult. In addition, many other factors such as preprocessing, the number of important attributes for the feature selection, the class imbalance have a significant effect on the assessment of To overcome these limitations, a model is proposed that includes the initial pre-processing step followed by the mandatory attribute selection and classification using association rule mining. In addition, the proposed model-based approach points in the right direction for research into the early detection of $\mathrm{AD}$ and has the potential to differentiate $\mathrm{AD}$ from healthy control subjects. The proposed methodology addresses pathologically proven data and overcomes class imbalance and overtraining problems. The proposed model is based on a single modality in order to overcome the higher computational costs and the combination of different modalities. They believe that pathologically tested data can increase accuracy and validity, while a balanced class helps classifiers provide accurate results. This model can help improve clinician predictive performance and address the limitations identified in previous research.

Noor et al. (Noor, 2020)supplied Application of deep learning in detecting neurological problems from magnetic resonance images: a survey at the detection of Alzheimer's ailment, Parkinson's ailment and schizophrenia. This article severely examines and compares performances of the prevailing deep learning (DL)-primarily based totally strategies to discover neurological problems - that specialize in Alzheimer's disease, Parkinson's ailment and schizophrenia-from MRI facts received the use of distinctive modalities along with practical and structural MRI. The comparative overall performance evaluation of numerous DL architectures throughout distinctive problems and imaging modalities indicates that the Convolutional Neural Network outperforms different strategies in detecting neurological problems. Advancement in high-velocity computing strategies and an extraordinary development with inside the improvement of novel DL-primarily based totally strategies and models open up precise possibility to are expecting and control some of neurological problems along with Alzheimer's disease, Parkinson's ailment and schizophrenia. In this paper, the maximum famous DL strategies had been explored in detecting the ones 3 main neurological problems from the MRI test facts.it summarizes pros, cons, and overall performance of those DL strategies for the neuroimaging facts.

In current years, the Deep Learning (DL) algorithms are very beneficial for the prognosis of AD as DL algorithms work nicely with huge datasets. Salehi A.W. et al.(Salehi, 2020) have applied Convolutional Neural Network (CNN) for the sooner prognosis and category of AD the usage of MRI snap shots, the ADNI three magnificence of images with the full quantity of 1512 mild, 2633 regular and $2480 \mathrm{AD}$ had been used. A big accuracy of $99 \%$ achieved wherein the model finished nicely as compared with many different associated works. Furthermore, evaluating the end result with preceding paintings on which ma-chine learning algorithms had been implemented the usage of OASIS dataset and it confirmed that after managing huge quantity of statistics like scientific statistics the deep learning strategies may be a higher alternative over the conventional machine learning techniques.

\section{PROPOSED SYSTEM}

\section{DATASET}

The dataset being used is obtained from OASIS which openly provides the relevant data containing cross-sectional MRI scans for demented and non-demented individuals ranging from the age of 18 years to 96 years. The dataset consists of 416 subjects, all of which are right handed. The dataset comprises of standardized T1-weighted Images; from which 100 subjects have mild to moderate dementia and 198 subjects which are all over the age of 60 .

\section{ARCHITECTURE}

The neural network consists of three convolution layer. Our first Convolution Layer starts with 32 filters, followed by a two Convolution Layer with 64 filters; each Convolution Layer is accompanied by a Pooling Layer (Max), All Convolution Layers and Pooling Layers were used with RELU as a primary activation function. After the final Convolution Layer-Pooling Layer, We propagate the results through a Final Layer. Softmax as the activation function in order to facilitate the classification done by the Final layer. And at the end, we iterated, optimized and trained our model with Adam optimizer. Adam Optimizer with learning rate 0.001 is used. Batch normalization was applied to the convolution layers in order to avoid the overfitting.

It is needed to optimize the error with a gradient descent optimizer. Adam optimizer is chosen instead of usual stochastic gradient descent algorithm, because of its adaptive nature. It computes adaptive learning rates for parameters from the $n-2$ and $\mathrm{n}-1$ moments of the gradient resulting in better optimization when compared to the stochastic method. And the data was split into test, train data randomly using cross validation.

Convolutional layer has set of learnable filters that may be carried out on enter images. Each operation calls for four hyper parameters:

$\mathrm{K}$, range of filters; Vector of weights to be carried out to input image,

F, their spatial extent; three Dimensions- width, top and depth.

$\mathrm{S}$, the dimensions of stride; Amount of displacement for the filter, in pixels.

$\mathrm{P}$, the quantity of (zero) padding; It manipulate dis-proportionate input to in shape the filter.

Table 2. Proposed CNN Layer

\begin{tabular}{|l|l|}
\hline 1 & Image Input \\
\hline 2 & Convolution \\
\hline 3 & Batch Normalization \\
\hline 4 & ReLU \\
\hline
\end{tabular}




\begin{tabular}{|l|l|}
\hline 5 & Max Pooling \\
\hline 6 & Convolution \\
\hline 7 & Batch Normalization \\
\hline 8 & ReLU \\
\hline 9 & Max Pooling \\
\hline 10 & Convolution \\
\hline 11 & Batch Normalization \\
\hline 12 & ReLU \\
\hline 13 & Max Pooling \\
\hline 14 & Fully Connected \\
\hline 15 & Softmax \\
\hline
\end{tabular}

\section{RESULT AND CONCLUSION}

In a paper using the convolution neural network to detect the Alzheimer disease with the same OASIS dataset got an accuracy of $80.25 \%$ (Ullah, 2018). But the model suggests the signal of overfitting which influences the overall performance of the version.

The performance of the method is evaluated on the basis of the metrics: accuracy, sensitivity and specificity. These metrics are defines as:

Accuracy $=\mathrm{TP}+\mathrm{TN} /$ total

Sensitivity $=\mathrm{TP} / \mathrm{P}$

Specificity $=\mathrm{TN} / \mathrm{N}$

Where:

$\mathrm{TP}$ is the value of true positive.

$\mathrm{TN}$ is the value of true negative.

$\mathrm{P}$ is the total number of positives.

$\mathrm{N}$ is total number of negatives.

The proposed method shows the accuracy of $82.3 \%$, sensitivity $=84.7 \%$, specificity $=81.2 \%$.

\section{Table. 1. Quantitaive results of the proposed method compared with other methods.}

\begin{tabular}{|l|l|}
\hline Tested method & Accuracy \\
\hline Proposed method & $82.3 \%$ \\
\hline HMT ULLAH(Ullah,2018) & $80.25 \%$ \\
\hline
\end{tabular}

The proposed model consist of three convolution layer each followed by the max pooling layer. ReLu is the primary activation function applied to the convolution layer. Softmax is the activation applied to the final layer. To avoid the overfitting of the model batch normalization was implemented to the each convolution layer. Adding batch normalization tends to cut back the inner covariate shift and instability in the distributions of the layer activation in deep neural networks, which reduces the result of overfitting. After the 545 epochs the model has shown the accuracy of $82.3 \%$. the model is smoother, and support the view that simpler networks are easier to train. A simple, smaller model also can go with the best results.

Not only for the detection of Alzheimers disease but also for the other diseases detection as brain tumor, breast cancer or heart failure diseases 3D MRI data plays a very important role as it can come up with the better results for the proper diagnosis of the particular disease. However, same approach can be applied for the detection of other diseases.

\section{FUTURE WORK}

Future research will attention on overall performance development of the CNN model.Maximum accuracy may be performed in a CNN through growing the quantity of data used to train the model. Here are some suggestions that may be useful for future systems.The different effects of each feature on the accuracy of the model can be analysed to optimize data input. Other work may include combining patient clinical data with imaging data to build the more reliable model and will improve the robustness of the model.

\section{REFERENCES}

[1] [1] Acharya, U.R., Fernandes, S.L., WeiKoh, J.E., Ciaccio, E.J., Fabell, M.K.M., Tanik, U.J., Rajinikanth, V. and Yeong, C.H., 2019. Automated detection of Alzheimer's disease using brain MRI images-a study with various feature extraction techniques. Journal of Medical Systems, 43(9), p.302.

[2] [2] Al-falluji, R.A.A., 2017. MRI based Techniques for Detection of Alzheimer: A Survey. International Journal of Computer Applications, 159(5), pp.20-24.

[3] [3] Allioui, H., Sadgal, M. and Elfazziki, A., 2019. Deep MRI segmentation: a convolutional method applied to Alzheimer disease detection. Int J Adv Comput Sci Appl, 10(11).

[4] [4] Convolutional Neural Networks: https://www.tensorflow.org/tutorials/

[5] [5] Farooq, A., Anwar, S., Awais, M. and Rehman, S., 2017, October. A deep CNN based multi-class classification of Alzheimer's disease using MRI. In 2017 IEEE International Conference on Imaging systems and techniques (IST) (pp. 1-6).IEEE.

[6] [6] Islam, J. and Zhang, Y., 2017, November. A novel deep learning based multi-class classification method for Alzheimer's disease detection using brain MRI data. In International Conference on Brain Informatics (pp. 213222).Springer, Cham.

[7] [7] Khan, A. and Usman, M., 2015, November. Early diagnosis of Alzheimer's disease using machine learning techniques: A review paper. In 2015 7th International Joint Conference on Knowledge Discovery, Knowledge Engineering and Knowledge Management (IC3K) (Vol. 1, pp. 380-387).IEEE.

[8] [8] LeCun, Y., Bottou, L., Bengio, Y. and Haffner, P., 1998. Gradient-based learning applied to document recognition. Proceedings of the IEEE, 86(11), pp.2278-2324.

[9] [9] Noor, M.B.T., Zenia, N.Z., Kaiser, M.S., Al Mamun, S. and Mahmud, M., 2020. Application of deep learning in detecting neurological disorders from magnetic resonance images: a survey on the detection of Alzheimer's disease, Parkinson's disease and schizophrenia. Brain informatics, 7(1), pp.1-21.

[10] [10] Patil, C., Mathura, M.G., Madhumitha, S., David, S.S., Fernandes, M., Venugopal, A. and Unnikrishnan, B., 2015, February. Using image processing on MRI scans. In 2015 IEEE International Conference on Signal Processing, Informatics, Communication and Energy Systems (SPICES) (pp. 1-5). IEEE.

[11] [11]Salehi, A.W., Baglat, P., Sharma, B.B., Gupta, G. and Upadhya, A., 2020, September. A CNN Model: Earlier Diagnosis and Classification of Alzheimer Disease using MRI. In 2020 International Conference on Smart Electronics and Communication (ICOSEC) (pp. 156-161).IEEE.

[12] [12] Toro, C.A.O., Sánchez, N.G., Gonzalo-Martín, C., Garcia, R.G., González, A.R. and Ruiz, E.M., 2019, June. Radiomics textural features extracted from subcortical structures of grey matter probability for Alzheimers disease detection. In 2019 IEEE 32nd International Symposium on Computer-Based Medical Systems (CBMS) (pp. 391-397).IEEE.

[13] [13] Ullah, H.T., Onik, Z., Islam, R. and Nandi, D., 2018, April. Alzheimer's disease and dementia detection from 3D brain MRI data using deep convolutional neural networks. In 2018 3rd International Conference for Convergence in Technology (I2CT) (pp. 1-3). IEEE.

[14] [14] Walokar, K., Khandelwal, M. and Bhingardive, A., 2012. Android Application for Dementia and Alzheimer's ManagementADAM.International Journal of Information and Electronics Engineering, 2(4), p.630. 


\section{AUTHORS}

First Author - Maskeen kaur, Master's Student, Baba Banda Singh Bahadur Engineering College, Fatehgarh Sahib (Punjab), India, maskeen.nahal@gmail.com
Second Author - Amanjot kaur, Professor (CSE)

Baba Banda Singh Bahadur Engineering College, Fatehgarh Sahib (Punjab), India, amanjot.kaur@bbsbec.ac.in 